\title{
Spinal Anesthesia for Amyotrophic Lateral Sclerosis Patient Undergoing Lower Extremity Orthopedic Surgery: An Overview of the Anesthetic Considerations
}

This article was published in the following Dove Press journal:

International Medical Case Reports Journal

Jason K Panchamia (D)

Carmelina Gurrieri

Adam W Amundson

Department of Anesthesiology and Perioperative Medicine, Mayo Clinic, Rochester, MN, USA
Correspondence: Jason K Panchamia Department of Anesthesiology and

Perioperative Medicine, Mayo Clinic, 200

First St SW, Rochester, MN 55905, USA

$\mathrm{Tel}+\mid$ 507-284-9698

$\mathrm{Fax}+$ I 507-284-0120

Email Panchamia.Jason@mayo.edu
Background: Amyotrophic lateral sclerosis is a progressive neurodegenerative disease primarily affecting the upper and lower motor neurons. Patients present with a variety of clinical manifestations inevitably resulting in death secondary to respiratory insufficiency from muscle weakness and consequential pulmonary complications. Despite the lack of universal consensus on the ideal anesthetic approach to amyotrophic lateral sclerosis patients undergoing lower extremity total joint surgery, there are few noteworthy anesthetic considerations in this cohort.

Case Report: A 75-year-old male with multiple medical comorbidities, including a recent diagnosis of amyotrophic lateral sclerosis, presented to a large academic medical center for a right total hip arthroplasty revision. The patient's preoperative neurologic examination demonstrated brisk deep tendon reflexes, visible fasciculations of lower extremities, and motor weakness of upper and lower extremities. Serology demonstrated an elevated creatine kinase, and an electromyography study showed active denervation in the cervical, thoracic, and lumbosacral regions. After a careful risk-benefit analysis was performed, involving a multidisciplinary team approach, the patient successfully underwent the surgical procedure with a spinal anesthetic and minimal sedation. Perioperative course was unremarkable, and there were no neurologic complications in the first 6 months after surgery.

Conclusion: Patients with amyotrophic lateral sclerosis present unique challenges for anesthesia providers. General anesthesia may potentially worsen respiratory function; therefore, alternative methods to avoiding airway manipulation should be considered. Conversely, regional techniques may carry the risk of exacerbating pre-existing neurologic symptoms. Currently, no definite guidelines exist on the perioperative anesthetic management of amyotrophic lateral sclerosis patients; ultimately, the decision to perform regional anesthesia should be based on analyzing a patient's risk against the potential benefits.

Keywords: arthroplasty, replacement, anesthesia, spinal, neurodegenerative diseases, risk assessment

\section{Introduction}

Amyotrophic lateral sclerosis (ALS), a subtype of motor neuron disease, is a progressive neurodegenerative disease that mainly affects motor neurons responsible for controlling voluntary muscle movement. ${ }^{1}$ Clinical manifestations are variable among patients due to location and onset of affected areas, rate of disease progression, and a mixture of upper motor neuron and lower motor neuron 
degeneration resulting in diverse signs and symptoms. ${ }^{2}$ Clinical features of ALS include progressive muscle weakness, muscular atrophy, fasciculations, spasticity, and hyperreflexia., ${ }^{3,4}$ ALS involvement of bulbar muscles can result in sialorrhea, dysphagia for solids and liquids, and dysarthria; patients typically require a percutaneous endogastric gastrostomy feeding tube to maintain nutrition intake. ${ }^{4}$ Respiratory-onset ALS is devastating, and patients may develop dyspnea on exertion, orthopnea, hypoventilation, and pneumonia; therefore, patients often require assisted breathing support devices such as noninvasive positive pressure ventilation, diaphragmatic pacing, or tracheotomy. ${ }^{3,4}$

Perioperative anesthesia management for ALS patients is challenging given the heterogeneous nature of the disease. ALS patients may be more sensitive to muscle relaxants and opioids resulting in postoperative respiratory failure, aspiration pneumonia, electrolyte abnormalities and hypovolemia due to poor nutrition intake, and exacerbation of neurologic symptoms and functional decline after surgery. ${ }^{3,5,6}$ Several large retrospective investigations have reported neuraxial anesthesia to improve perioperative outcomes compared to general anesthesia in patients undergoing lower extremity joint replacement surgery. ${ }^{7-9}$ However, there is no consensus on the perioperative management for ALS patients undergoing lower extremity orthopedic surgery because there is a paucity of data due to the rarity and short life expectancy of the disease. ${ }^{10}$ Therefore, ALS patients present a unique set of circumstances which require a detailed risk versus benefit analysis of regional and general anesthetic techniques on a case by case basis. We present a case of a patient with ALS who successfully underwent a spinal technique as a primary anesthetic for a total hip revision. The patient and his wife (caretaker and power of attorney) provided written consent to review and report this case, and the Mayo Clinic Institutional Review Board approved this project.

\section{Case Presentation}

A 75-year-old male (American Society of Anesthesiologists physical status, class III; height, $176 \mathrm{~cm}$; weight, $94 \mathrm{~kg}$; body mass index, 30) presented for right total hip arthroplasty revision due to severe pain. His medical history was significant for diabetes mellitus type II (on insulin and metformin), hyperlipidemia, obstructive sleep apnea (non-compliant with CPAP therapy), and slowly progressive dementia (managed with donepezil; his caretaker is his wife). With respect to his dementia, the patient was diagnosed with mild cognitive impairment 6 years before surgery after taking the Montreal Cognitive Assessment test; however, his symptoms had gradually progressed to a moderate cognitive decline prior to surgery.

The following details the sequence of events: 1 year prior to surgery, his wife first noticed a change in the patient's gait described as shuffling steps. Subsequently, 5 months prior to surgery, the patient started complaining of pain in his thighs and difficulty standing up from a seated position, and 2 months prior to surgery, the patient experienced several falls attributed to frequent tripping. A neurologist was consulted a few days prior to surgery for further evaluation. Physical exam revealed diffusely brisk deep tendon reflexes in upper and lower extremities (Mayo Clinic scale for tendon reflex assessment -4 to $+4{ }^{11}+1$ (brisk) for biceps, brachioradialis, triceps, quadriceps with crossed adductor response, and Achilles reflexes) with negative Hoffman sign, Babinski sign absent on right and equivocal on left, and visible fasciculations of right external rotators, right deltoid, bilateral gastrocnemius and quadriceps muscles. Motor testing demonstrated mild motor weakness of upper (Medical Research Council scale for muscle power 0 to 5 , Right Side/Left Side: external rotators $4 / 4$, deltoid $4 / 5$, biceps $4 / 5$, triceps $4 / 5$, wrist extension $4 / 5$, wrist flexion $5 / 5$, finger extension $4 / 4$, interossei $4 / 4$, abductor pollicis brevis $4 / 5$ ) and lower extremities (Medical Research Council scale for muscle power 0 to 5 , Right Side/Left Side: Iliopsoas $4 / 4$, thigh adductor $5 / 5$, thigh abductors $5 / 5$, quadriceps $5 / 4$, hamstrings $5 / 5$, tibialis anterior $5 / 5$, gastrocsoleus $5 / 5$, foot inversion $5 / 5$, foot eversion $4 / 4$, toe extension $4 / 4$, toe flexion 4/4). Sensory testing was notable for mildly decreased vibration and pinprick sensation in great toes. During gait testing, the patient was slow to stand up, unable to stand on his heels, and was walking with a slight wide-based stance with steppage on the left leg. The patient denied any problems with breathing, chewing, or swallowing. His speech and language were normal, palate elevation was symmetric, and there were no tongue fasciculation. Serology demonstrated an elevated creatine kinase levels up to $1825 \mathrm{U} / \mathrm{L}$ (reference level 39-308 U/ L). Electromyography study showed neurogenic changes with active denervation in the cervical, thoracic, and lumbosacral segments. Magnetic resonance imaging revealed normal morphology of the spinal cord, conus medullaris, and cauda equina with mild to moderate cervical 
spondylosis and foraminal stenosis. Head computed tomography taken during evaluation for a recent fall showed mild brain parenchymal volume loss. Electrocardiogram demonstrated sinus bradycardia which was unchanged from a previous electrocardiogram 13 years prior. Given these findings, the patient's clinical and pathological features were highly suspicious for ALS. Of note, he had a hip replacement 10 years ago at an outside hospital with no reported complications; however, the patient was unable to recall the type of anesthetic he received.

On the day of surgery, the patient appropriately fasted for at least 8 hours, and his vital signs were heart rate 97, blood pressure $152 / 93$, oxygen saturation $96 \%$, and respiratory rate 20 . After a multidisciplinary team discussion involving the surgical and anesthesia team, the patient elected to have spinal anesthesia due to potential concerns of postoperative respiratory failure associated with ALS. In the operating room, an 18-gauge IV was established, and standard anesthetic monitoring was applied (5-lead electrocardiogram, blood pressure, pulse-oximeter, temperature, and capnography), and intravenous midazolam $(2 \mathrm{mg})$ and fentanyl $(50 \mathrm{mcg})$ were administered for sedation during the spinal procedure. A 1-liter fluid bolus of lactated ringers was administered during the regional anesthesia procedure. The patient was positioned in the lateral decubitus position, and an intrathecal injection of $1.8 \mathrm{~mL}$ of $0.75 \%$ isobaric bupivacaine was administered using a 22-gauge, 3.5-inch Whitacre needle at L3-L4 via midline approach under sterile conditions. During the surgical procedure the patient received mild sedation with a propofol infusion (10 to $30 \mathrm{mcg} / \mathrm{kg} / \mathrm{min}$ ) titrated to a Richmond Agitation-Sedation Scale of -1 , and the patient was spontaneously breathing with a nasal cannula for oxygen support. Intravenous dexamethasone (4 mg), cefazolin (2 g), tranexamic acid ( $2 \mathrm{~g})$, and ephedrine (25 mg in divided doses) were also administered intraoperatively. The surgeon performed a peri-articular block using a ropivacaine solution for postoperative analgesia. ${ }^{12,13}$ Surgery was uneventful with a total procedure time of approximately 2 hours. The patient remained hemodynamically stable (heart rate 60 to 70 , respiratory rate 14 to 16 , oxygen saturation $100 \%$, non-invasive blood pressure mean 65 to 80 , skin temperature $36^{\circ} \mathrm{C}$ ) during the intraoperative period with minimal fluctuation in vitals for the exception of periodic low blood pressure readings which was responsive to small doses of ephedrine. In the recovery unit, the patient had minimal pain and he did not experience nausea. He was discharged from the recovery unit within 1 hour of arrival, and his vital signs prior to discharge was heart rate 78 , blood pressure 158/86, respiratory rate 14 , oxygen saturation $98 \%$ on room air, temperature $36.4{ }^{\circ} \mathrm{C}$. Postoperatively, the patient was able to slowly ambulate and participate in physical therapy (Activity Measure for Post-Acute Care Inpatient Short Form during hospitalization: Basic Mobility Raw Score increased from 14 to 18, and Daily Activities Raw Score increased from 16 to 17). He was discharged from the hospital on postoperative day 7 to a skilled nurse facility for ongoing rehabilitation. No neurological complications were noted during his hospitalization and at a 6-month follow-up.

\section{Discussion}

ALS is a progressive neurodegenerative disease primarily affecting the upper and lower motor neurons. Death generally occurs from respiratory insufficiency due to debilitating muscle weakness. Our case describes a patient suspicious for limb-onset ALS, likely in the early stages because his ALS clinical manifestations were limited to the muscles of the lower extremity. Currently, there is no universal agreement on the ideal anesthetic technique (regional versus general anesthesia) for ALS patients undergoing lower extremity total joint replacement. Further, there is no information guiding anesthetic management in patients with early versus advanced clinical features, as well as different phenotype presentations (eg, limb-onset versus bulbar-onset) of this fatal disease. ${ }^{4,10}$

Some major anesthetic concerns in this patient population include autonomic dysregulation, gastric aspiration, postoperative respiratory failure, and increased sensitivity to opioids and sedative agents. We applied a cautious approach to our anesthetic plan for two reasons: 1) the lack of robust clinical trials evaluating safety outcomes in ALS patients undergoing different anesthesia techniques, and 2) the risk of sub-clinical symptoms of respiratory, bulbar, or autonomic nervous system involvement, which could be exacerbated during the perioperative period (eg, anesthetic medications, acute blood loss, upregulation of inflammatory markers from surgery). Additional testing was not performed because the patient required surgery regardless due to his severe pain and diminished quality of life; further, additional work-up would not preclude his surgery. Accordingly, our anesthetic goals entailed minimizing hemodynamic perturbations, limiting opioid consumption, avoiding use of neuromuscular blocking agents, and avoiding airway manipulation. In a team discussion 
involving the patient, family, anesthesia, and surgical teams, it was decided that a spinal anesthetic with mild sedation would be the best option to achieve the anesthetic goals for this patient.

Large databases comparing regional anesthesia to general anesthesia for lower extremity surgery are limited to patients with no pre-existing central nervous system (CNS) disorders. Previous investigations report regional anesthesia to be associated with lower 30-day mortality, shorter hospital length of stay, decreased transfusion rate and overall reduced perioperative morbidity. ${ }^{7-9}$ In contrast, the impact of general anesthesia on pulmonary function should be strongly considered in ALS patients given the progressive weakness affecting the respiratory muscles inherent to the destructive pathogenesis of the disease. ${ }^{14}$ Other complications of ALS patients undergoing general anesthesia include aspiration pneumonia, residual muscle paralysis requiring ventilator support after rocuronium reversal with Sugammadex, and life-threatening hyperkalemia after succinylcholine administration., ${ }^{3,15,16}$ In a recent case series from our institution, Hoeper et al reported general anesthesia related complications in a cohort of 78 ALS patients, including four cases of prolonged postoperative intubation related to pre-existing respiratory muscle weakness, one case of post-extubation respiratory distress requiring tracheal re-intubation, and four cases of prolonged hospital stay. ${ }^{3}$

There are several important risks to consider in ALS patients undergoing spinal anesthetics. The degree of autonomic nervous system dysfunction parallels the natural progression of the disease, and can present as vagal withdrawal with sympathetic predominance, vasomotor instability, or sympathetic denervation with vagal predominance. ${ }^{17}$ Initiation of spinal anesthesia can result in local sympathectomy and vasodilation, both contributing to hypotension. Further, high spread of local anesthetics can result in blockade of the T1-T4 cardioaccelerator fibers, resulting in hypotension and bradycardia with ensuing cardiovascular collapse. In this scenario, spinal induced hypotension can theoretically be exacerbated by ALS associated autonomic dysfunction. To minimize this, we provided one liter of intravenous fluid in the beginning of the case and we utilized an isobaric local anesthetic solution, which spreads in the subarachnoid space unrelated to position and gravity. Hyperbaric solutions can also be administered; however, careful patient position is crucial to preventing further cranial spread as the solution is dependent on gravity and curvature of the spine in any horizontal position. ${ }^{18}$ Further, vasoactive medications and invasive monitoring (eg, arterial line) should be readily available to treat and monitor hemodynamic instability, respectively. Conduction abnormalities can occur in ALS patients; ${ }^{17}$ therefore, a preoperative EKG is valuable, and at least 5-lead EKG monitoring is essential during the perioperative period.

Spinal anesthesia is commonly performed with administration of analgesics and anxiolytics during the block procedure to help alleviate pain and anxiety, respectively, and titration of sedation agents during the intraoperative period under monitored anesthesia care. Moderate anesthesia care can have deleterious consequences in ALS patients with poor pulmonary reserve and/or in patients who are unable to adequately clear secretions. Specifically, previous studies have reported cases of aspiration pneumonia, respiratory distress requiring prolonged mechanical ventilation, and high risk of 30-day mortality during moderate anesthesia care. ${ }^{19,20}$ We administered a propofol infusion at a dose which provided mild sedation and permitted spontaneous respiration, purposeful feedback to tactile and verbal stimulation, and did not require airway manipulation (especially important given his predilection to airway obstruction due to obstructive sleep apnea). ${ }^{21}$ It would be prudent to have the patient awake or mildly sedated since higher doses of propofol can result in loss of airway and gastric reflexes, in addition to impaired cardiovascular and ventilatory function consequently exposing ALS patients to increased risk of complications. $^{21}$

Though rare, nerve injury after spinal anesthesia can occur in the general population through various mechanisms, some of which include mechanical trauma from inadvertent needle penetration of neural tissue, nerve ischemia from epinephrine induced vasoconstriction, compression of spinal cord from epidural hematoma or volume of epidural injectate, and chemical nerve injury such as local anesthetic neurotoxicity. Patients with pre-existing CNS disorders can potentially be at increased risk for nerve injury or experience exacerbation of their preoperative neurologic symptoms due to a "double-crush" phenomenon where patients with previous neural compromise may be prone to a secondary insult from the aforementioned mechanisms. ${ }^{22-24}$

Current literature evaluating the safety of regional anesthesia in ALS patients is conflicting. Dripps and Vandam reported a case series of 11 patients with preexisting neurologic diseases, including ALS, who 
experienced worsening neurologic symptoms after undergoing spinal anesthesia. ${ }^{5}$ Similarly, Kane et al reported a series of neurologic sequelae after spinal and epidural anesthesia. ${ }^{25}$ More recently, Hara et al described a case of a successful lumbar epidural anesthesia for inguinal herniorrhaphy in a 69-year-old patient with ALS complicated by a transient postoperative decrease in vital capacity. ${ }^{14}$ Other cases of uneventful neuraxial anesthesia have been described in ALS patients with no associated neurologic complications. ${ }^{23,26}$ Finally, Hebl et al investigated a large series of 139 patients with pre-existing CNS disorders, including 5 cases of ALS, who underwent neuraxial anesthesia and analgesia. None of the patients had new or worsening postoperative neurologic deficits during follow-up when compared with preoperative findings. ${ }^{24}$ Despite these new encouraging data on regional anesthesia in patients with ALS, no definite consensus on the use of regional anesthesia currently exists. Of note, the neurologic complications reported several decades ago were primarily related to pre-existing spinal stenosis, spinal cord tumors, and specific local anesthetics concentrations and preparation of the injectate solution. ${ }^{5,25,27}$ Moreover, identifying the etiology of postoperative neurologic deficits is often complex because other contributing factors include body habitus, tourniquet inflation pressure, improper patient positioning, and prolonged or difficult labor. ${ }^{24}$ Recently, surgical stress has been associated with postoperative inflammatory neuropathy irrespective of anesthetic management. ${ }^{28,29}$ Overall, the possibility of postoperative neurologic deterioration should be considered in patients with ALS and weighed against the risk of worsening respiratory failure after general anesthesia.

In conclusion, we reported an uneventful case of spinal anesthesia in a patient with recent diagnosis of ALS undergoing a lower extremity arthroplasty procedure. Although no definite recommendations can be given based on this case report, spinal anesthesia can be an alternative option for ALS patients undergoing lower extremity orthopedic procedures. Ultimately, the decision to use regional anesthesia should be individualized on a case-by-case basis involving a thorough risk-benefit analysis, establishing goals for safe perioperative management, and initiating a multidisciplinary discussion between the patient, anesthesia, surgery, and neurology teams. Further prospective studies are needed to make definitive conclusions on the safety of regional anesthesia techniques in this highrisk patient population.

\section{Abbreviations}

ALS, amyotrophic lateral sclerosis; CNS, central nervous system.

\section{Ethics Approval and Consent to Participate}

This project was approved by the Mayo Clinic Institutional Review Board.

\section{Consent for Publication}

Written informed consent for publication of the clinical details was obtained from the patient.

\section{Author Contributions}

All authors participated in the design, execution, and/or analysis of the work presented, attests to the accuracy and validity of the contents, took part in drafting, revising or critically reviewing the article; gave final approval of the version to be published; have agreed on the journal to which the article has been submitted; and agree to be accountable for all aspects of the work.

\section{Disclosure}

The authors declare that they have no competing interests in this work.

\section{References}

1. Onders R. Amyotrophic lateral sclerosis: the Midwestern surgical experience with the diaphragm pacing stimulation system shows that general anesthesia can be safely performed. Am J Surg. 2009;197 (3):386-390. doi:10.16/j.amjsurg.2008.1011.1008

2. Creemers H, Grupstra H, Nollet F, van den Berg LH, Beelen A. Prognostic factors for the course of functional status of patients with ALS: a systematic review. $J$ Neurol. 2015;262(6):1407-1423. doi:10.1007/s00415-00014-07564-00418

3. Hoeper A. Amyotrophic lateral sclerosis and anesthesia: a case series and review of the literature. $J$ Anesth. 2019;33(2):257-265. doi:10.1007/s00540-019-02611-x

4. Zarei S, Carr K, Reiley L, et al. A comprehensive review of amyotrophic lateral sclerosis. Surg Neurol Int. 2015;6:171. doi:10.4103/ 2152-7806.169561

5. Dripps RD, Vandam LD. Exacerbation of pre-existing neurologic disease after spinal anesthesia. $N$ Engl J Med. 1956;255(18):843-849. doi:10.1056/NEJM195611012551803

6. Pinto S, Swash M, de Carvalho M. Does surgery accelerate progression of amyotrophic lateral sclerosis? J Neurol Neurosurg Psychiatry. 2014;85(6):643-646. doi:10.1136/jnnp-2013-305770

7. Wilson JM, Farley KX, Erens GA, Guild GN3rd. General vs spinal anesthesia for revision total knee arthroplasty: do complication rates differ? J Arthroplasty. 2019;34(7):1417-1422. doi:10.1016/j.arth.201 9.03 .048

8. Memtsoudis SG, Sun X, Chiu YL, et al. Perioperative comparative effectiveness of anesthetic technique in orthopedic patients. Anesthesiology. 2013;118(5):1046-1058. doi:10.1097/ALN.0b013e318286061d 
9. Perlas A, Chan VW, Beattie S. Anesthesia technique and mortality after total hip or knee arthroplasty: a retrospective, propensity score-matched cohort study. Anesthesiology. 2016;125(4):724-731. doi:10.1097/ALN.0000000000001248

10. Hardiman O, Van Den Berg LH, Kiernan MC. Clinical diagnosis and management of amyotrophic lateral sclerosis. Nat Rev Neurol. 2011;7 (11):639-649. doi:10.1038/nrneurol.2011.153

11. Manschot S, van Passel L, Buskens E, Algra A, van Gijn J. Mayo and NINDS scales for assessment of tendon reflexes: between observer agreement and implications for communication. J Neurol Neurosurg Psychiatry. 1998;64(2):253-255. doi:10.1136/jnnp.64.2.253

12. Amundson AW, Johnson RL, Abdel MP, et al. A three-arm randomized clinical trial comparing continuous femoral plus single-injection sciatic peripheral nerve blocks versus periarticular injection with ropivacaine or liposomal bupivacaine for patients undergoing total knee arthroplasty. Anesthesiology. 2017;126 (6):1139-1150. doi:10.1097/ALN.0000000000001586

13. Panchamia JK, Amundson AW, Jacob AK, et al. A 3-arm randomized clinical trial comparing interscalene blockade techniques with local infiltration analgesia for total shoulder arthroplasty. J Shoulder Elbow Surg. 2019;28(10):e325-e338. doi:10.1016/j.jse.2019.05.013

14. Hara K, Sakura S, Saito Y, Maeda M, Kosaka Y. Epidural anesthesia and pulmonary function in a patient with amyotrophic lateral sclerosis. Anesth Analg. 1996;83(4):878-879. doi:10.1097/ 00000539-199610000-199600040

15. Turner M, Lawrence H, Arnold I, Ansorge O, Talbot K. Catastrophic hyperkalaemia following administration of suxamethonium chloride to a patient with undiagnosed amyotrophic lateral sclerosis. Clin Med (Northfield Il). 2011;11(3):292-293. doi:10.7861/clinmedicine.11-3-292

16. Chun HR, Chung J, Kim NS, Kim AJ, Kim S, Kang KS. Incomplete recovery from rocuronium-induced muscle relaxation in patients with amyotrophic lateral sclerosis using sugammadex: a case report. Medicine (Baltimore). 2020;99(3):e18867. doi:10.1097/MD.0000000000018867

17. Shemisa K, Kaelber D, Parikh SA, Mackall JA. Autonomic etiology of heart block in amyotrophic lateral sclerosis: a case report. $J$ Med Case Rep. 2014;8:224. doi:10.1186/1752-1947-8-224

18. Hocking G, Wildsmith JA. Intrathecal drug spread. Br J Anaesth. 2004;93(4):568-578. doi:10.1093/bja/aeh204

19. Thompson AG, Blackwell V, Marsden R, et al. A risk stratifying tool to facilitate safe late-stage percutaneous endoscopic gastrostomy in ALS. Amyotroph Lateral Scler Frontotemporal Degener. 2017;18(3-4):243-248. doi:10.1080/21678421.21672016.21274330
20. Sato Y, Goshi S, Kawauchi Y, et al. Safety of unsedated PEG placement using transoral ultrathin endoscopy in patients with amyotrophic lateral sclerosis. Nutr Neurosci. 2017;20(1):71-75. doi:10.1179/1476830514Y.0000000161

21. Apfelbaum J, Gross JB, Connis RTet al,. Practice Guidelines for Moderate Procedural Sedation and Analgesia 2018: A Report by the American Society of Anesthesiologists Task Force on Moderate Procedural Sedation and Analgesia, the American Association of Oral and Maxillofacial Surgeons, American College of Radiology, American Dental Association, American Society of Dentist Anesthesiologists, and Society of Interventional Radiology. Anesthesiology. 2018;128(3):437-479. doi:10.1097/ ALN.0000000000002043

22. Kopp S. Regional anesthesia in patients with preexisting neurologic disease. Reg Anesth Pain Med. 2015;40(5):467-478. doi:10.1097/ AAP.0000000000000179

23. Hobaika AB, Neves BS. Combined spinal-epidural block in a patient with amyotrophic lateral sclerosis: case report. Rev Bras Anestesiol. 2009;59(2):206-209. doi:10.1590/S0034-70942009000200008

24. Hebl JR, Horlocker TT, Schroeder DR. Neuraxial anesthesia and analgesia in patients with preexisting central nervous system disorders. Anesth Analg. 2006;103(1):223-228. doi:10.1213/01. ane.0000220896.56427.53

25. Kane RE. Neurologic deficits following epidural or spinal anesthesia. Anesth Analg. 1981;60(3):150-161. doi:10.1213/00000539198103000-00006

26. Kock-Cordeiro. Combined spinal-epidural anesthesia with non-invasive ventilation during cesarean delivery of a woman with a recent diagnosis of amyotrophic lateral sclerosis. Int $J$ Obstet Anesth. 2018;36:108-110. doi:10.1016/j.ijoa.2018.06.001

27. Chen, Chen LK, Chang Y, Liu CC, Hou WY. Epidural anesthesia combined with propofol sedation for abdominal hysterectomy in a patient with amyotrophic lateral sclerosis-a case report. Acta Anaesthesiol Sin. 1998;36(2):103-106.

28. Ahn KS, Kopp SL, Watson JC, Scott KP, Trousdale RT, Hebl JR. Postsurgical inflammatory neuropathy. Reg Anesth Pain Med. 2011;36(4):403-405. doi:10.1097/AAP.0b013e31821e6503

29. Staff NP, Engelstad J, Klein CJ, et al. Post-surgical inflammatory neuropathy. Brain. 2010;133(10):2866-2880. doi:10.1093/brain/ awq252
International Medical Case Reports Journal

\section{Publish your work in this journal}

The International Medical Case Reports Journal is an international, peer-reviewed open-access journal publishing original case reports from all medical specialties. Previously unpublished medical posters are also accepted relating to any area of clinical or preclinical science. Submissions should not normally exceed 2,000 words or 4 published pages including figures, diagrams and references. The manuscript management system is completely online and includes a very quick and fair peer-review system, which is all easy to use. Visit http://www.dovepress.com/testimonials.php to read real quotes from published authors. 